\title{
Highly flexible absolute integrated encoder system on GMR-basis
}

\author{
Burkhard Stritzke, Christoph Brode, Marcus Danowski \\ Lenord, Bauer \& Co. GmbH \\ Dohlenstrasse 32 \\ 46145 Oberhausen \\ bstritzke@lenord.de \\ cbrode@lenord.de \\ mdanowski@lenord.de
}

\begin{abstract}
:
The cooperative project GMR-Flex is funded by the Federal Ministry of Education and Research (BMBF) within the support program "Microsystems 2004-2009". The aim of the GMR-Flex project is the development of a GMR-based absolute rotary encoder system, which is flexibly scalable between module 0.2 and 4 and shaft diameters from 20 to $500 \mathrm{~mm}$. One of the project's primary objectives is an accuracy of 14 bits, which has solely been achieved by optical encoder systems up to now. Furthermore, the system should be easily parameterizable and mountable
\end{abstract}

Key words: GMR-Flex, Encoder Kit, Nonius Interpolation, Toothed Wheel, Hollow Shaft

\section{Introduction}

Concerning the control of electrical drives and servo motors a major challenge is the exact detection of the rotor position and feedback to the motor controller which can mainly be accomplished by optical encoders today.

In contrast to optical encoders whose measurement of the angular position is severely disturbed or often impossible under harsh environmental conditions, absolute magnetic encoder systems offer reliable solutions.

The known magnetic-based encoder systems with integrated bearings currently have to be adapted with high effort to the shaft diameter and the measuring scales of each drive system. Another disadvantage of assembled absolute magnetic encoder systems is the relatively small shaft diameter which can only be adapted with high effort.

The advantages of magnetic-based angle measuring systems are well known. Their quality is the possibility of full encapsulation of the scanning unit which leads to resistance to water, oils, greases and other aggressive media. Furthermore the ferromagnetic target wheels or code disks feature a high resistance to mechanical shock and vibration.

There is a substantial difference between encoder kits and standard encoders. The scanning unit is built up of a sensor and a toothed wheel or a code disc which are mounted separately. The responsibility for keeping the required mechanical precision in design and mounting falls to the customer. Cause to this fact the system's accuracy increases significantly.

People often neglect that couplings or torque supports are required while using a standard encoder with integrated bearings which leads to overload of the bearings and reduced accuracy. In addition important parameters in the selection of the bearings are temperature, regarding grease variety, speed, mechanical stress and requirements of impermeability. Another point to note is the limited durability of the bearings.

A universal use of standard encoders is complicated, expensive and inflexible due to the fact that depending on the application a different bearing design has to be chosen if necessary.

The MiniCoder-series GEL244 which has been established on the market for high speed motor spindles is a prime example for successful use of modular encoders. A compact design, bearingless speed measurement and flexible design of target wheels are the advantages that can be shown by the contactless working measurement unit. 
The implementation of bearing design for highspeed spindles occurs only once in the application. The encoder supplier does not need to solve the same problem for the encoder.

Other advantages of the MiniCoder-series are:

- minimum space low initial costs related to the adaption of the target wheel or shaft assembly, inner and outer diameter

- small number of variants of the encoder for a huge number of different target wheels

- logistic advantage respecting the number of variants for the end customer

Yet there are encoders in incremental versions with $\sin /$ cos-signals and reference signals with different period lengths of target wheel. Based on his specific needs in application concerning resolution, variation of the air gap etc. the costumer has to make a selection. This decision is highly time-consuming as the number of options supplied by $L+B$ target wheels is considerably more than 400 .

A trend towards direct drive and torque motors exists for quite a long time and still remains. Higher dynamics and precision, lower wear and the compact design are the main reasons for this trend.

A large shaft diameter and a compact design are usually characteristics for direct drives, its applications are mainly used in harsh industrial environments. These applications often require determining the position instantly when the machine is turned on. Due to the demand of high torsional moment right from the moment of starting the machine, reference position search routines are not allowed. For this reason an absolute measuring equivalent of incremental MiniCoder-series is necessary.

\section{Targets}

- Fully encapsulated absolute measuring system with small dimensions, which can be integrated easily and with low parametrization and assembly costs.
- The target wheel or the code disk need to be flexible in the dimensioning of the inner and outer diameter. To be economically efficient even for small quantities the initial effort for design and production must be low, independent of the inner and outer diameter of the hollow shaft.

\section{Theory and Concept}

The joint project's goal is to close the technological gap between rotary encoders with integrated bearings and incremental encoders by developing an absolute encoder. The demand for a flexible, scalable encoder system made it necessary to investigate new technologies.

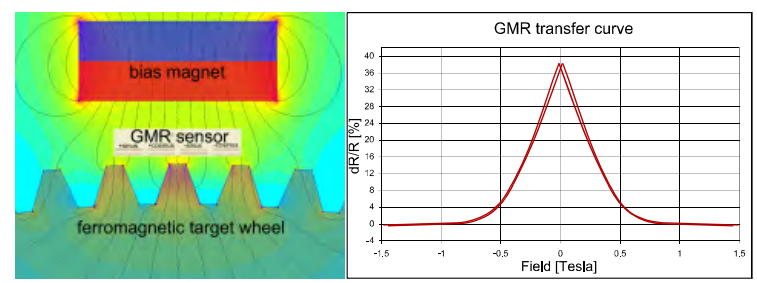

Figure 1: Principle of GMR sensor with back bias magnet and target wheel (left), typical GMR transfer curve (right)

The basic technology of the encoder system forms the back-bias technology in which the integrated bias magnet generates the magnetic field of the GMR-sensor. In combination with high-precision toothed wheels sin/cos signals of high quality will be generated (see figure 1).

The intended total system accuracy of up to 14 bits is implemented by using a new approach of two-track nonius analysis shown in figure 2 . Previously, such accuracies of incremental magnetic systems have not been possible.

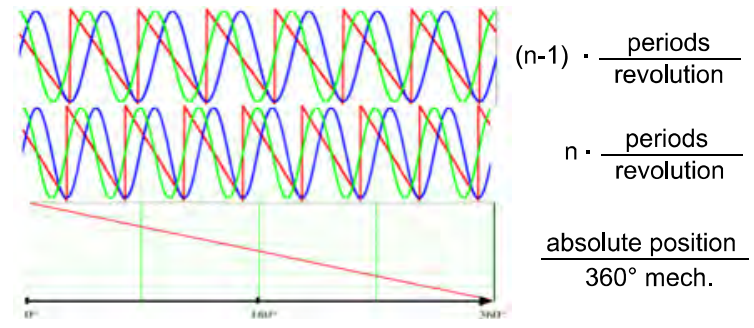

Figure 2: Principle of two-track nonius analysis

The flexibility of the encoder system is now achieved by two different approaches. On the one hand by adapting the GMR sensor layouts and on the other hand by a variable number of teeth of the toothed wheel or code disc.

An important component of the encoder system is the interpolation ASIC that has been developed by the GEMAC $\mathrm{mbH}$. The 
interpolation ASIC increases the flexibility of the total system by implementing innovative features. The integration of various common interfaces in the ASIC allows to use the system in many applications.

The general concept of the GMR-based encoder is shown in figure 3.

\section{Realization}

The first results at the beginning of the project showed, that increasing the number of teeth is only useful up to a certain extent. The accuracy of the tooth position is a physical limit, the number of teeth of the nonius system is restricted and the scanning diameter cannot be indefinitely increased.

To increase the achievable hollow shaft diameter the tooth period length has consequently to be increased without degrading the accuracy of tooth position. This is a new technological requirement, because there is no GMR sensor technology available that allows such a high accuracy or the possibility to interpolate such long tooth periods.

The back-biased magnetic assembly and the sensor system have been simulated and optimized to elaborate a signal quality which is independent of mechanical positioning tolerances.
As a result the manufacturing method of target wheels is relevant to define tooth shapes, which produce a magnetic field modulation as sinusoidal as possible. To use the established manufacturing process of target wheels two approaches have been pursued. First, the GMR Flex sensor technology was designed in a way that even a non-sinusoidal modulation magnetic field provides a sinusoidal output signal. This was realized by a special design of the GMR strip layout. The second approach will be described in section "Nonius interpolation-ASIC GC-NIP"

The challenge for Sensitec and $L+B$ was to design a GMR sensor layout with tooth period lengths up to a length of $6-7 \mathrm{~mm}$ that show identical accuracy to the already existing shorter GMR sensor layouts. Results of simulation and measurements of many different produced toothed wheels showed a fundamental difference in defect characteristics. The shorter period length is limited in accuracy due to stochastic defect characteristics. The results for the large tooth period lengths showed systematic defect characteristics. This remaining defect can be corrected with help of a certain algorithm in the ASIC.

In the test lab it was demonstrated that both approaches allow an expansion of the existing limit of nonius from $32 \mid 31$ teeth to $128 \mid 127$ teeth with a clear increase of the scanning diameter.

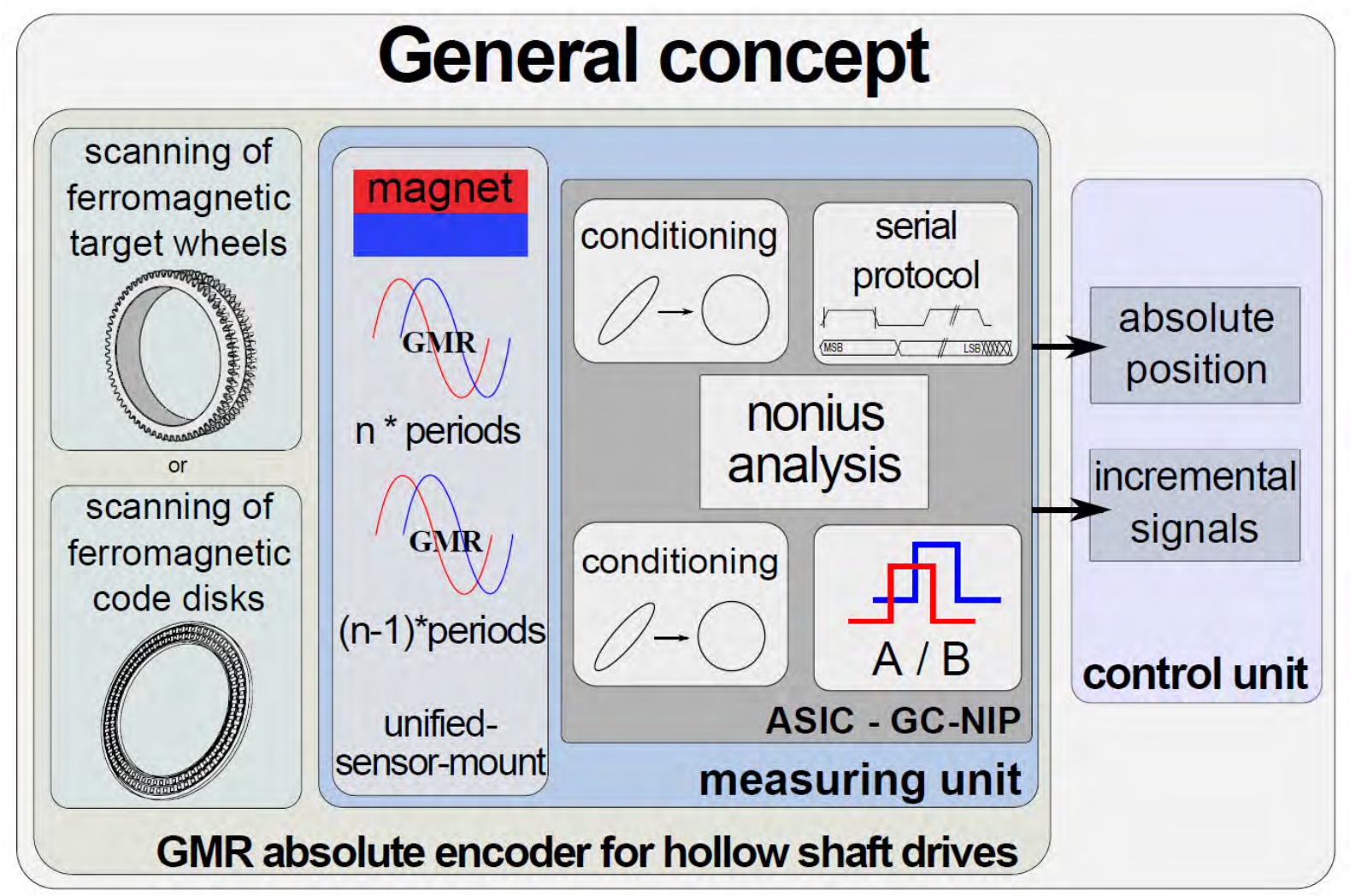

Figure 3: General concept of the GMR-Flex-project 


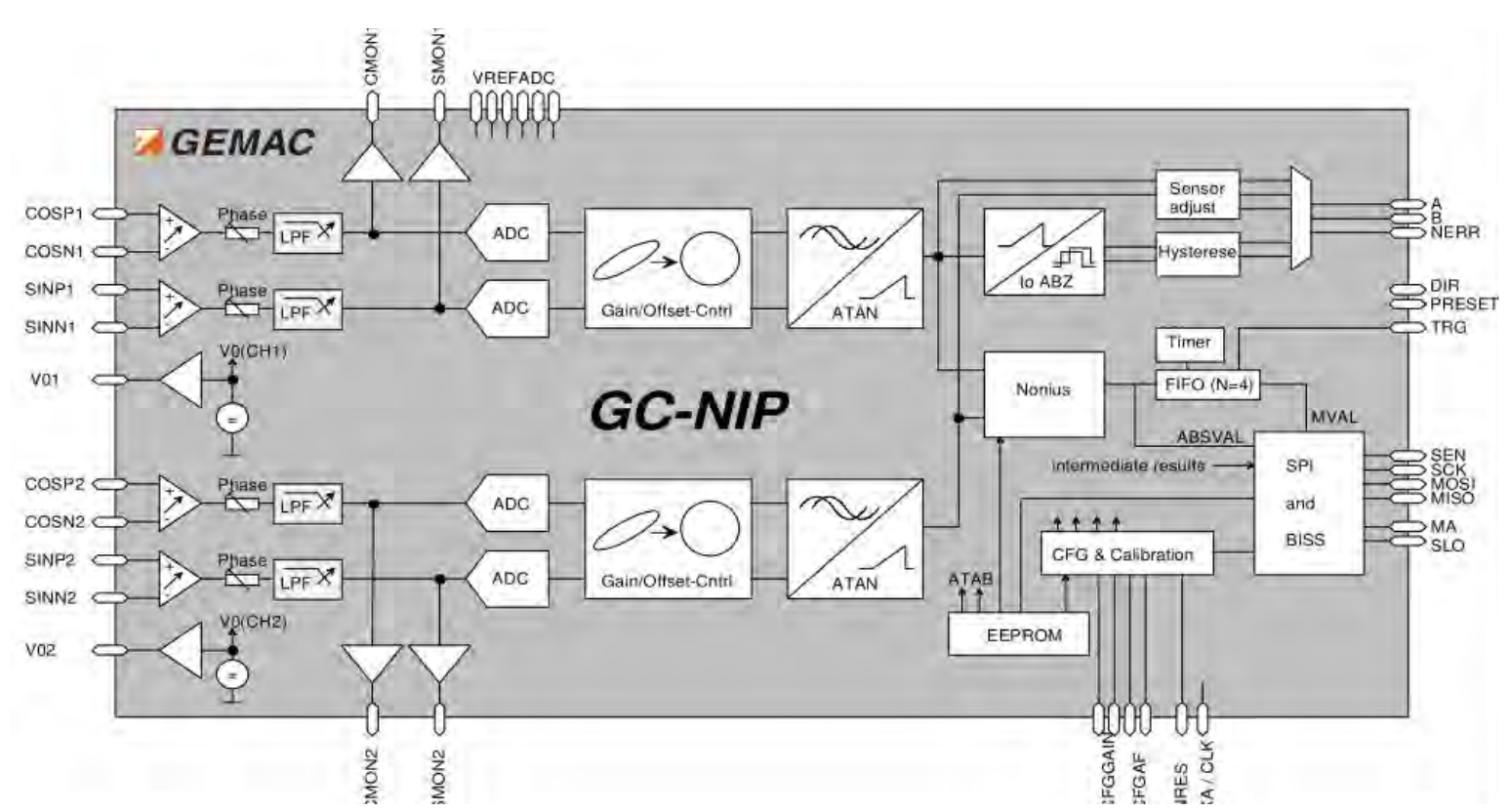

Figure 4: Block diagram of interpolation ASIC GC-NIP

In comparison this result represents an increase of the possible scanning diameter for a two-channel nonius system by a factor of 16 .

\section{Dimensioning the GMR-Flex ferromagnetic targets}

Due to the flexibility of the encoder systems and the unique packaging a fast and simple adaption to different constructions is feasible. Up to now there is no reason of time consuming changes concerning the scanning unit by changing the measuring scale. To cover a wide range of applications for the absolute angle measurement it is necessary, that the required space of the scanning unit is as small as possible and that the design of the target wheel permits a wide range of inner and outer diameter.

\section{Nonius interpolation-ASIC GC-NIP}

Until now the evaluation systems available on the market are only able to convert binary numbers of teeth like $8|7,16| 15,32 \mid 31$ to an absolute angle information. This makes a continuous scaling of the scanning diameter impossible because the scanning diameter has to be doubled when the period length of the target wheel stays constant. This leads to an inflexible system. Therefore the new nonius ASIC offers a free choice of teeth combinations in a wide range.

Due to the patented regulation of offset and amplitude of the company GEMAC a manual signal conditioning is unnecessary. This simplifies the manufacturing and assembly costs considerably. The new developed algorithm improves the overall system accuracy by correcting the systematic errors. The Principle of this algorithm is shown in figure 5.

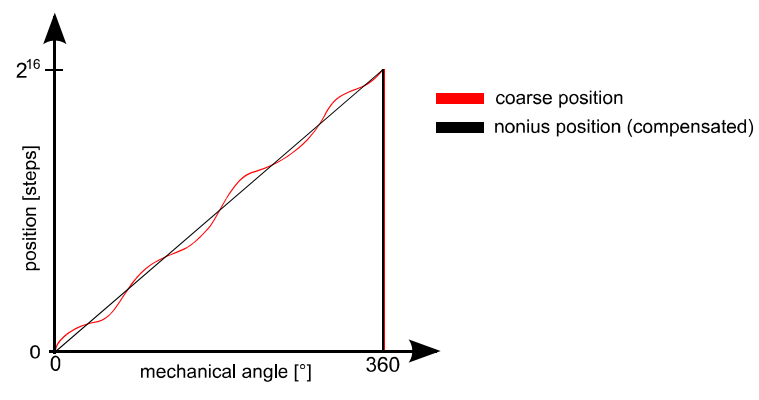

Figure 5: Principle of compensation of the remaining error

The high level of integration, the small number of additional external circuitry and the small dimensions of the IC packages allow for a small product size, which results in a high constructive degree of freedom for the integration of the encoder system.

By integrating standard interfaces such as SSI and quadrature signals, as well as innovative and efficient new interfaces such as BISS interface, an adaption to many applications is possible. The block diagram of the new developed interpolation ASIC GC-NIP is shown in figure 4.

\section{Results}

The requirement of a flexible system from module 0.2 to module 4 made the development 
of new GMR sensors necessary. In close collaboration with Sensitec four new monolithic GMR gradiometer layouts have been designed. These four designs cover the range up to module 2.

With a precise placement of single bridge elements in free scalable pitch GMR gradiometers a module up to 4 has been realized. To obtain sin/cos-signals of high quality, all modules have been designed with a geometric filter like shown in figure 5.

The results for harmonic distortion for all new designed GMR gradiometer sensors is below $0.5 \%$ in a wide range of temperature and air gap.

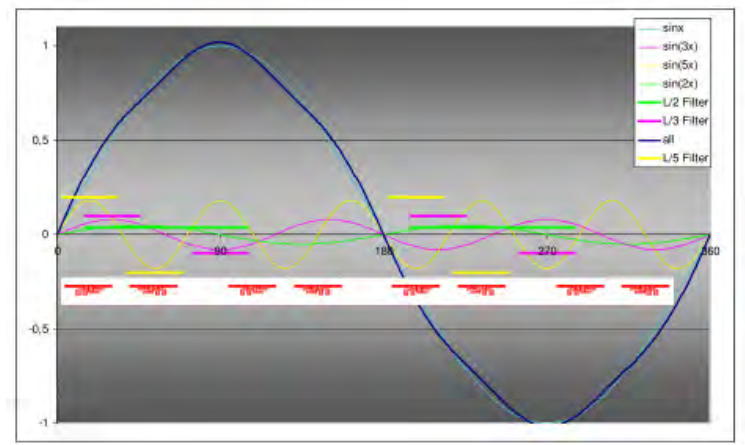

Figure 6: Schematic representation of the GMR sensors with geometric filter

To realize a unique mounting process of the various GMR sensors to the PCB a chip carrier with a uniform footprint has been realized as shown in figure 7. Thereby it is possible to place the different variations of the GMR sensors without changing the layout of the PCB.

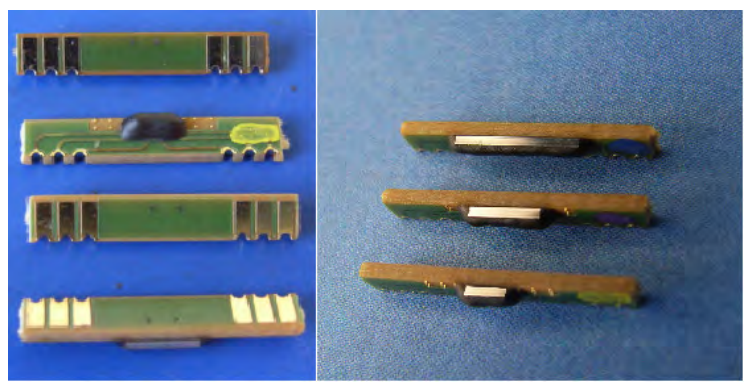

Figure 7: Unified-sensor-mounts for various GMR sensors

In the course of the project a new tooling machine for the production of nonius toothed wheels has been qualified for series production.
As examples a small number of variants is shown in figure 8.

With another production process established in series at Lenord + Bauer since 2005 the flexible design of nonius code discs is available. An example of a two-tracked nonius code disc is also shown in figure 8.
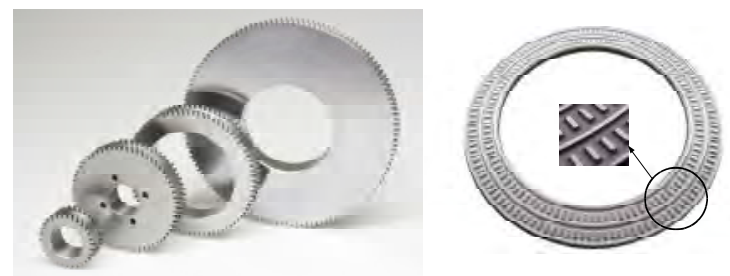

Figure 8: Nonius toothed wheels with various inner and outer diameter and same module (left), twotracked nonius code disc (right)

\section{Measurements and test results}

The test results show robustness against mechanical tolerances and allow use in harsh environments. The results of the project partner Harmonic Drive show no influence on the accuracy and function of GMR sensors in the immediate vicinity of a magnetic brake and motor winding.

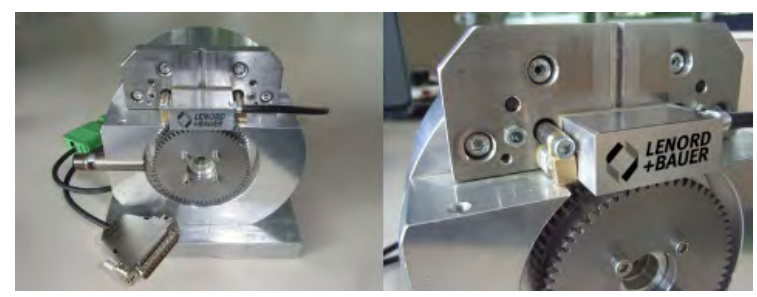

Figure 9: Test setup for nonius encoder kit

By using large tooth period lengths large air gaps are possible. This is beneficial for magnetic bearing drives in large hollow shafts and in case of drive overload. The selfcalibration allows a quick and easy installation at the end customer. Also it offers the measurement of the system reserve and robustness.

The absolute accuracy could be enhanced to more than 13 bit by the use of high-precision target wheels with large modules in combination with the developed GMR sensors by Sensitec. The resolution may reach up to 20 bits with a high degree of repeating accuracy. The design and adaptation of the system to changing installation situations can be implemented easily. 


\begin{tabular}{|c|c|c|c|c|c|}
\hline module & 0.2 & 0.4 & 1 & 2 & $2-4$ \\
\hline chip type & $\begin{array}{c}\text { monolithic } \\
\text { chip }\end{array}$ & $\begin{array}{l}\text { monolithic } \\
\text { chip }\end{array}$ & $\begin{array}{l}\text { monolithic } \\
\text { chip }\end{array}$ & $\begin{array}{l}\text { monolithic chip / } \\
\text { multiple placement of } \\
\text { single elements }\end{array}$ & $\begin{array}{c}\text { multiple placement of } \\
\text { single elements }\end{array}$ \\
\hline
\end{tabular}

\begin{tabular}{|c|c|c|c|c|c|}
\hline $\begin{array}{l}\text { hollow shaft } \\
\text { range }\end{array}$ & $<40 \mathrm{~mm}$ & $\begin{array}{l}\text { up to } \\
40 \mathrm{~mm}\end{array}$ & $\begin{array}{c}\text { up to } \\
100 \mathrm{~mm}\end{array}$ & up to $200 \mathrm{~mm}$ & up to $500 \mathrm{~mm}$ \\
\hline
\end{tabular}

Figure 10: Tooth period, GMR-gradiometer and hollow shaft diameter

\section{Conclusions and perspectives}

The goal of the project "GMR-Flex" funded by the Federal Ministry for Education and Research was to create a basic technology to integrate an absolute magnetic encoder system in large hollow shaft applications. It was able to achieve a fast and flexible integration useable in various industries, complementary with a very high precision.

With the flexibility of the concept and the characteristics of high-precision toothed wheel production also in small quantities a fast implementation with minimal effort is possible. Low installation and programming costs increase the customers acceptance. A maintenance-free, robust and precise absolute encoder on GMR technology can thus be found in many new applications.

Main applications are located in key industries such as mobile equipment, electric vehicles, marine propulsion systems and railway technology.

First positive tests of the technology took place at the projects partner Harmonic Drive and a quick implementation is planned. In addition there are many other customer requests to be addressed with help of the new encoder system.

The basic technology of high-precision measurement of large and small tooth period lengths by GMR sensors expands the range of applications of GMR technology. This allows a dynamic and high-precision motion detection and can contribute to the demand of energysaving and increasing efficiency in many industries.

The company $L+B$ is grateful to the Federal Ministry of Education and Research for funding this important project.

\section{References}

[1] J. Traute, U. Loreit: "Abtastung von weichmagnetischen Zahnstrukturen mit MRSensoren", Vorträge zum Symposium, 2005

[2] F. Dettmann, U.Loreit: "Grundlagen magnetoresistiver Sensoren”, Vorträge zum Symposium, 1992

[3] B. Stritzke, "Flexible Scalable GMR-based Rotary Encoder System for Hollow Shaft Drives (GMRFlex)", mst-news No. 4/2009

[4] T. Bausch, Innovative Zahnradfertigung, expert verlag, 2006

[5] Sensitec GmbH, Application Note GLM700 Familie, 2012

[6] H. Grimm, Internal release, 2010

[7] M. Danowski, B. Stritzke (Lenord, Bauer \& Co. $\mathrm{GmbH})$, R. Schmidt, T. Sichting (Elbau Elektronik Bauelemente GmbH Berlin), EP 1816440 B1, 2008

[8] IBM Corporation, Engineering \& Technology Services, EMEA E\&TS, Sensor Design for a magnetic bridge, 2003 